\title{
Remoção de fósforo em lagoas de polimento operadas em regime de bateladas sequenciais
}

\section{Phosphorus removal in polishing ponds operated under sequential batch regime}

Data de entrada: 02/06/2019

Data de aprovação: $18 / 06 / 2020$

Isabella Vieira Santos ${ }^{1}$ | Silvânia Lucas dos Santos² | Adrianus van Haandel ${ }^{3 *}$

DOI: https://doi.org/10.36659/dae.2021.022

ORCID ID

Santos IV (iD https://orcid.org/0000-0003-1111-8146
Santos SL (iD https://orcid.org/0000-0002-6905-3966 Haandel A https://orcid.org/0000-0002-9937-6715

\section{Resumo}

Neste artigo, apresenta-se um modelo para a remoção de fósforo em um robusto sistema de tratamento de esgoto que alia o tratamento anaeróbio em um reator UASB ao pós-tratamento em lagoas de polimento, operadas em regime de batelada sequencial (LPBS). Os processos que ocorrem nas LPBS favorecem um aumento no valor do $\mathrm{pH}$, o que leva à remoção de fósforo pela precipitação de fosfato na presença de íons de cálcio. Foi possível obter uma reduzida concentração de fósforo $\left(<1 \mathrm{mgP} . \mathrm{L}^{-1}\right)$ em uma água moderadamente dura ( $\left.1 \mathrm{mmolCa} . \mathrm{L}^{-1}\right)$ quando o $\mathrm{pH}$ na lagoa atingiu valores superiores a 9,5. Constatou-se que o fósforo precipitava nas lagoas na forma de hidroxiapatita de cálcio $\mathrm{C}_{10}(\mathrm{OH})_{2}\left(\mathrm{PO}_{4}\right)_{6}$. Assim, foi demonstrada a viabilidade da remoção do fósforo no sistema UASB-LPBS, sem a necessidade de insumos (produtos químicos, por exemplo) ou energia, utilizando apenas a energia solar.

Palavras-chave: Salubridade ambiental. PMSB. ISA.

\section{Abstract}

This article presents a model of phosphorus removal in a new sewage treatment system, consisting of anaerobic treatment in a UASB reactor and post-treatment in polishing ponds operated in a sequential batch regime (LPBS). The processes that take place in the LPBS cause a $\mathrm{pH}$ increase, which in turn leads to the removal of nutrients by phosphate precipitation in the presence of calcium ions. It was possible to obtain very low concentrations of phosphorus $\left(<1 \mathrm{mgP.L}^{-1}\right)$ when the $\mathrm{pH}$ in the pond reached values above 9.5. It was established that phosphorus precipitated in the lagoons in the form of calcium hydroxyapatite $\mathrm{C}_{10}(\mathrm{OH})_{2}\left(\mathrm{PO}_{4}\right)_{6}$. Thus, the feasibility of removing phosphorus in the UASB-LPBS system was demonstrated, without the need for inputs of materials or energy, except for solar energy.

Keywords: Removal of nutrients. Precipitation. Calcium phosphate.

\footnotetext{
1 Universidade Estadual da Paraíba - UEPB - Campina Grande - Paraíba - Brasil.

${ }^{2}$ Universidade Federal do Rio Grande do Norte - UFRN - Natal - Rio Grande do Norte - Brasil.

${ }^{3}$ Universidade Federal de Campina Grande - UFCG - Campina Grande - Paraíba - Brasil.

* Autor correspondente: adrianusvhagmail.com.
} 


\section{INTRODUÇÃO}

As lagoas de estabilização são amplamente utilizadas nos países em desenvolvimento, por apresentarem simplicidade operacional e baixo custo para a construção (CASAROTTI et al., 2012). Embora sejam eficientes para reduzir as concentrações de sólidos suspensos e do material orgânico, estas pouco atuam na remoção de nutrientes. Cavalcanti (2003) demonstrou que é necessário um aumento do $\mathrm{pH}$ para que uma fração considerável dos nutrientes nitrogênio e fósforo seja removida. Em relação ao fósforo, Reed et al. (1995) mostraram que o mesmo é passível de ser removido eficientemente por mecanismos físicos (precipitação) quando o pH está acima de 9, o que não é observado em lagoas de estabilização convencionais. Von Sperling et al. (2009) também afirmaram que o $\mathrm{pH}$ deve ser superior a 9 para que haja remoção considerável desse nutriente, apesar de o fosfato ser termodinamicamente instável para valores de $\mathrm{pH}$ acima de 7.

Essa deficiência de remoção dos nutrientes constitui uma das grandes desvantagens do uso desse sistema de tratamento, visto que a grande maioria dos efluentes é lançada em corpos de água e, quando estes possuem quantidades elevadas de nutrientes, podem contribuir para acelerar o processo de eutrofização, deteriorando a qualidade da água. No caso dos sistemas de lagoas de estabilização, o efluente tratado geralmente não apresenta qualidade, principalmente em termos de nutrientes, tal que permita a descarga em águas superficiais dentro dos limites impostos pela legislação, especificamente a Resolução do Conselho Nacional do Meio Ambiente (Conama) n 357/2005 e n 430/2011 (BRASIL, 2005, 2011).

O mecanismo básico de aumento do $\mathrm{pH}$ em lagoas é a remoção biológica de dióxido de carbono, que ocorre quando a taxa de remoção pela fotossíntese é maior que a taxa de produção de material orgânico (SANTOS; VAN HAANDEL, 2021).
Todavia, o critério básico de projetos de lagoas de estabilização convencionais leva em consideração o estabelecimento de condições para que haja um equilíbrio entre a fotossíntese e a oxidação do material orgânico, visando evitar que a lagoa se torne anaeróbia (PARKER, 1950). Nessas condições, o aumento do $\mathrm{pH}$ não se materializa e a remoção de nutrientes não ocorre.

A combinação do pré-tratamento anaeróbio (reator UASB) com lagoas de polimento surge como uma alternativa satisfatória em substituição ao uso do sistema de lagoas de estabilização convencional. Reatores do tipo UASB apresentam elevada eficiência no tratamento de esgoto, removendo entre $65 \%$ a $80 \%$ da matéria orgânica (VAN HAANDEL; LETTINGA, 1994), de modo que a concentração residual e, consequentemente, a taxa de oxidação, seja baixa. Além disso, possuem a vantagem de remover parte considerável dos sólidos em suspensão, produzindo um efluente com baixa turbidez e boa transparência, favorecendo uma alta taxa de fotossíntese. 0 desequilíbrio entre a alta taxa de fotossíntese e a baixa taxa de oxidação do material orgânico é a força indutora do consumo de dióxido de carbono e, portanto, do aumento do pH no pós-tratamento do efluente do reator UASB em uma lagoa.

$\mathrm{O} \mathrm{pH}$ elevado nas lagoas de polimento favorece a remoção de nutrientes. No caso do nitrogênio amoniacal, o alto $\mathrm{pH}$ desloca o equilíbrio de dissociação e aumenta a concentração de amônia gasosa dissolvida que é volátil e pode ser removida por dessorção (MORAIS; SANTOS; VAN HAANDEL, 2021). $O$ aumento do $\mathrm{pH}$ resulta também em uma mudança de equilíbrio das espécies de fosfatos, aumentando a concentração do íon PO4-3 que, na presença de cátions como Cálcio (Ca) ou Magnésio ( $\mathrm{Mg}$ ) (que geralmente se encontram no esgoto), pode precipitar na forma de sais de fosfato. 
$\mathrm{Na}$ Tabela 1, apresentam-se alguns dos sais de fosfato com os valores da razão molar entre os íons de cálcio e de fosfato (razão $\mathrm{Ca} / \mathrm{P}$ ), suas respectivas fórmulas químicas e as constantes de solubilidade (Kps).

Tabela 1 - Sais de fosfato, dissociações, composição e constantes de solubilidade $\left(\mathrm{K}_{\mathrm{ps}}\right)$

\begin{tabular}{|c|c|c|c|}
\hline Sais de fosfato & Reação de dissociação & $\begin{array}{c}\text { Relação Teórica de } \\
\text { Ca/P }\end{array}$ & $\begin{array}{l}\text { Constante } \mathrm{K}_{\mathrm{ps}} \\
\left(20-25^{\circ} \mathrm{C}\right)^{\mathrm{ps}}\end{array}$ \\
\hline Fosfato de Hidroxicálcico & $\mathrm{Ca}_{2} \mathrm{HPO}_{4}(\mathrm{OH})_{2}(\mathrm{~s}) \leftrightarrow 2 \mathrm{Ca}^{+2}+\mathrm{HPO}_{4}^{-2}+2 \mathrm{OH}^{-}$ & 2 & $10^{-22.6}$ \\
\hline Fosfato de Octacálcio & $\mathrm{Ca}_{4} \mathrm{H}\left(\mathrm{PO}_{4}\right)^{3} \cdot 3 \mathrm{H}_{2} \mathrm{O}(\mathrm{s}) \leftrightarrow 4 \mathrm{Ca}^{+2}+3 \mathrm{PO}_{4}^{-3}+\mathrm{H}^{+}+3 \mathrm{H}_{2} \mathrm{O}$ & 1,33 & $10^{-48}$ \\
\hline Fosfato Tricálcico Amorfo & $\mathrm{Ca}_{3}\left(\mathrm{PO}_{4}\right)_{2}(\mathrm{~s}) \leftrightarrow 3 \mathrm{Ca}^{+2}+2 \mathrm{PO}_{4}^{-3}$ & 1.5 & $10^{-25.2}$ \\
\hline Fosfato Dicálcico Di-hidratado & $\mathrm{CaHPO}_{4} \cdot 2 \mathrm{H}_{2} \mathrm{O}(\mathrm{s}) \leftrightarrow \mathrm{Ca}^{+2}+\mathrm{HPO}_{4}^{-2}+2 \mathrm{H}_{2} \mathrm{O}$ & 1 & $10^{-25.2}$ \\
\hline Hidroxiapatita de cálcio & $\mathrm{Ca}_{5}(\mathrm{OH})\left(\mathrm{PO}_{4}\right)^{3}(\mathrm{~s}) 5 \mathrm{Ca}^{+2}+3 \mathrm{PO}_{4}^{-3}+\mathrm{OH}^{-}$ & 1,67 & $10^{-58.5}$ \\
\hline
\end{tabular}

Tendo em vista que a concentração de fósforo no esgoto é da ordem de $10 \mathrm{mgP.L^{-1 }}$ (JORDÃO;

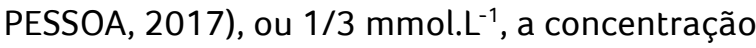
de Ca necessária para que haja a precipitação estequiométrica de fosfatos de cálcio deve ser da ordem de $1 / 2$ a 2/3 mmol. L-1 ou de 20 a $25 \mathrm{mgCa} \cdot \mathrm{L}^{-1}$. Essa concentração normalmente é encontrada no esgoto doméstico bruto.

A precipitação de fosfato de cálcio só é possível se houver condições termodinamicamente favoráveis. Assim, por exemplo, para a precipitação de hidroxiapatita de cálcio (HA) tem-se (Eq. 1):

$\mathrm{Ca}_{5}(\mathrm{OH})\left(\mathrm{PO}_{4}\right)_{3}(\mathrm{~s}) \leftrightarrow 5 \mathrm{Ca}^{+2}+3 \mathrm{PO}_{4}^{-3}+\mathrm{OH}^{-}$

A expressão do equilíbrio para essa reação é apresentada na Eq. 2, com a sua respectiva constante de solubilidade. Para os casos em que o produto iônico da equação seja maior que Kps, a precipitação do fosfato será possível.

$\mathrm{K}_{\mathrm{ps}}=\left\{\mathrm{Ca}^{+2}\right\} 5\left\{\mathrm{PO}_{4}^{-3}\right\} 3\left\{\mathrm{OH}^{-}\right\}=1 \times 10^{-58,5} \mathrm{~mol} \cdot \mathrm{L}^{-1}$

As principais condições que influenciam a precipitação do fosfato de cálcio são $\mathrm{pH}$, temperatura, concentrações de fosfato e de cálcio presentes no efluente e a duração da precipitação. Em lagoas de polimento, o pH é afetado pela profundidade e pelo regime hidrodinâmico. $O$ tipo de operação das lagoas é outro fator determinante para a remoção de nutrientes (SANTOS; VAN HAANDEL, 2021). Albuquerque, Santos e van Haandel (2021) mostraram que lagoas de polimento, quando operadas em regime de fluxo contínuo (LPFC), não removem o fósforo eficiente e confiavelmente, visto que o $\mathrm{pH}$ nessas lagoas geralmente não se eleva suficientemente. Bastos et al. (2007), por sua vez, ao avaliar a remoção de fósforo em lagoas de polimento (LPFC) com profundidades variando de 0,3 a 0,9 m também não conseguiram obter remoções consideráveis de fósforo, mesmo para elevados tempos de detenção hidráulica (TDH > 18 dias), o maior percentual de remoção alcançado foi de $33,2 \%$.

Em contraste, mostra-se no presente artigo que as lagoas de polimento quando operadas em regime hidráulico de batelada sequencial (LPBS) permitem o estabelecimento de um $\mathrm{pH}$ mais elevado do que as lagoas de polimento de fluxo contínuo (LPFC), favorecendo assim, a remoção do fósforo presente por precipitação. No presente artigo, apresentamse os resultados de uma investigação experimental sobre a remoção de fósforo em lagoas de polimento em escala piloto, operadas em regime de bateladas sequenciais. Foram observadas a influência da profundidade das lagoas, das condições climáticas e do TDH sobre essa remoção. 
1.1 Modelo de equilíbrio termodinâmico para precipitação do fosfato

O modelo matemático aqui descrito representa uma ferramenta de simulação, pela qual se estima a forma do sal de fosfato que precipitou nas lagoas de polimento, bem como, a concentração de fosfato residual. A modelagem foi realizada por meio de equações, considerando as possíveis formas de sais de fosfato encontradas no esgoto doméstico, bem comofoi avaliada a influência exercida por alguns parâmetros, especificamente o $\mathrm{pH}$, sobre a precipitação do fósforo.

Existem as seguintes formas de fósforo, dependendo do $\mathrm{pH}: \mathrm{PO}_{4}{ }^{3-}$ (fosfato), $\mathrm{H}_{2} \mathrm{PO}_{4}^{-}$(dihidrogenofosfato), $\mathrm{HPO}_{4} 2^{-}$(hidrogenofosfato) e $\mathrm{H}_{3} \mathrm{PO}_{4}$ (ácido fosfórico). Os equilíbrios entre estas formas são:

$\mathrm{H}_{3} \mathrm{PO}_{4} \leftrightarrow \mathrm{H}_{2} \mathrm{PO}_{4}^{-}+\mathrm{H}^{+}$

$\left[\left(\mathrm{H}_{2} \mathrm{PO}_{4}^{-}\right)\left(\mathrm{H}^{+}\right)\right] / \mathrm{H}_{3} \mathrm{PO}_{4}=\mathrm{K}_{1}$

$\left[\mathrm{H}_{3} \mathrm{PO}_{4}\right]=\left[\mathrm{H}_{2} \mathrm{PO}_{4}^{-}\right]\left[\mathrm{H}^{+}\right] / \mathrm{K}_{1}$

$\mathrm{H}_{2} \mathrm{PO}_{4}^{-} \leftrightarrow \mathrm{HPO}_{4}^{-2}+\mathrm{H}^{+}$

$\left[\left(\mathrm{HPO}_{4}^{-2}\right)\left(\mathrm{H}^{+}\right)\right] / \mathrm{H}_{2} \mathrm{PO}_{4}^{-}=\mathrm{K}_{2}$

$\left[\mathrm{H}_{2} \mathrm{PO}_{4}^{-}\right]=\left[\mathrm{HPO}_{4}^{-2}\right]\left[\mathrm{H}^{+}\right] / \mathrm{K}_{2}$

$\mathrm{HPO}_{4}^{-2} \leftrightarrow \mathrm{PO}_{4} 3^{-}+\mathrm{H}^{+}$

$\left[\left(\mathrm{PO}_{4}^{-3}\right)\left(\mathrm{H}^{+}\right)\right] / \mathrm{HPO}_{4}^{-2}=\mathrm{K}_{3}$

$\left[\mathrm{HPO}_{4}^{-2}\right]=\left[\mathrm{PO}_{4}^{-3}\right]\left[\mathrm{H}^{+}\right] / \mathrm{K}_{3}$

As constantes de dissociação a $25^{\circ} \mathrm{C}$ são: $\mathrm{pK}_{1}=2,23 \mathrm{pK}_{2}=7,21 \mathrm{pK}_{3}=12,32$.

De posse dessas equações, pode-se calcular a concentração total de todas as espécies de ortofosfato com a seguinte equação:

$\mathrm{C}_{\mathrm{t}}=\left[\mathrm{H}_{3} \mathrm{PO}_{4}\right]+\left[\mathrm{H}_{2} \mathrm{PO}_{4}^{-}\right]+\left[\mathrm{HPO}_{4}{ }^{2-}\right]+\left[\mathrm{PO}_{4}{ }^{3-}\right]$
$\mathrm{Ou}$

$\mathrm{C}_{\mathrm{t}}=\left[\mathrm{PO}_{4}{ }^{3-}\right]\left(1+\left[\mathrm{H}^{+}\right] / \mathrm{K}_{3}+\left[\mathrm{H}^{+}\right]^{2} /\left(\mathrm{K}_{2} \mathrm{~K}_{3}\right)+\left[\mathrm{H}^{+}\right]^{3} /\left(\mathrm{K}_{1} \cdot \mathrm{K}_{2} \cdot \mathrm{K}_{3}\right)(7)\right.$

Portanto:

$\left[\mathrm{PO}_{4} 3^{-}\right]=\mathrm{C}_{\mathrm{t}} /\left(1+\left[\mathrm{H}^{+}\right] / \mathrm{K}_{3}+\left[\mathrm{H}^{+}\right]^{2} /\left(\mathrm{K}_{2} \mathrm{~K}_{3}\right)+\left[\mathrm{H}^{+}\right]^{3} / \mathrm{K}_{1} \cdot \mathrm{K}_{2} \cdot \mathrm{K}_{3}\right)(8)$

A partir de então, foi calculada a concentração de quatro formas de sais de fosfato de cálcio em função do $\mathrm{pH}$ para os valores das constantes de solubilidade termodinâmica $\left(\mathrm{K}_{\mathrm{ps}}\right)$ apresentadas na Tabela 1. Adotou-se uma concentração de $1 \mathrm{mmol} . \mathrm{L}^{-1}$ de cálcio, isto é, uma água moderadamente dura, como é o caso da água que compõe o esgoto de Campina Grande, na Paraíba.

- Para o fosfato de Octacálcio: $\left[\mathrm{Ca}^{+2}\right]^{4}\left[\mathrm{PO}_{4}^{-3}\right]^{3}$ $\left[\mathrm{H}^{+}\right]=10^{-48}$

- Para a hidroxiapatita: $\left[\mathrm{Ca}^{+2}\right]^{5}\left[\mathrm{PO}_{4}^{-3}\right]^{3}\left[\mathrm{OH}^{-}\right]=$ $10^{-58.5}$

- Para o Fosfato tricálcico amorfo: $\left[\mathrm{Ca}^{+2}\right]^{3}\left[\mathrm{PO}_{4}^{-3}\right]^{2}=$ $10^{-25.2}$

- Para o Fosfato Dicálcico Di-hidratado: $\left[\mathrm{Ca}^{+2}\right]^{2}$ $\left[\mathrm{PO}_{4}^{-3}\right]\left[\mathrm{H}^{+}\right]=10^{-25.2}$

Após a realização dos cálculos das concentrações das formas de fosfatos de cálcio, foi plotado um diagrama de solubilidade termodinâmica para esses sais em função do pH (Fig. 1). No entanto, sabe-se que a solubilidade na prática pode ser muito maior que a solubilidade termodinâmica da Fig. 1, especialmente na presença de material orgânico, como invariavelmente é o caso em lagoas de polimento (CAPRI; MARAIS, 1974). Portanto, antecipa-se que a solubilidade nas lagoas será maior do que a termodinâmica prevê. 


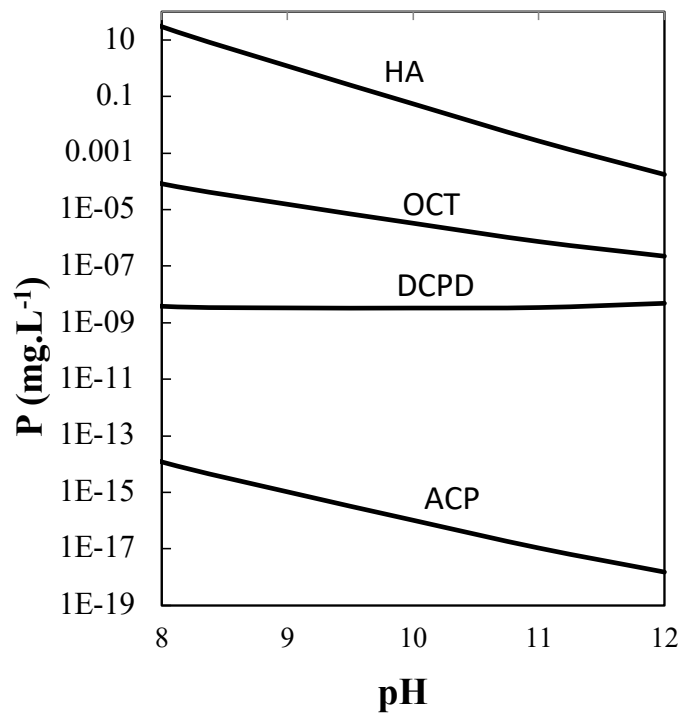

\section{Legenda:}

ACP - Fosfato tricálcico amorfo;

OCP - Fosfato de Octacálcio;

HA - Hidroxiapatita;

DCPD - Fosfato Dicálcico Di-hidratado;

Figura 1 - Diagrama da solubilidade termodinâmica para cinco minerais fosfatados e sua relação com o pH $\left(\mathrm{Ca}=1 \mathrm{mmol} . \mathrm{L}^{-1}\right)$.

\section{METODOLOGIA}

\subsection{Descrição do sistema}

Realizou-se uma investigação experimental em que a viabilidade da remoção de fosfato, mediante aumento do $\mathrm{pH}$ em lagoas de polimento recebendo efluente de reatores UASB, foi analisada. A pesquisa foi desenvolvida na cidade de Campina Grande - PB, no Nordeste do Brasil. O sistema de tratamento consistiu em um reator UASB para a produção de esgoto digerido e um conjunto de 4 lagoas de polimento, em escala piloto, com profundidades distintas; ambas foram operadas em regime de bateladas sequen- ciais (LPBS). O reator UASB era alimentado com esgoto municipal.

As LPBS, construídas em fibra de vidro, tinham forma cilíndrica com diâmetro de $0,5 \mathrm{~m}$ e profundidades de 0,2; 0,4; 0,6 e 1,0 m, conforme apresentado na Fig. 2. Para uniformizar o conteúdo da fase líquida e evitar a flotação de algas, a superfície das lagoas era agitada suavemente por uma palheta de $1 \mathrm{~cm}$ de largura, acionada por um motor com rotação de $6 \mathrm{rpm}$. Parte do efluente do reator UASB era direcionado, de forma descontínua, para as lagoas de polimento. A Fig. 3 mostra um esquema de todo o sistema de tratamento.

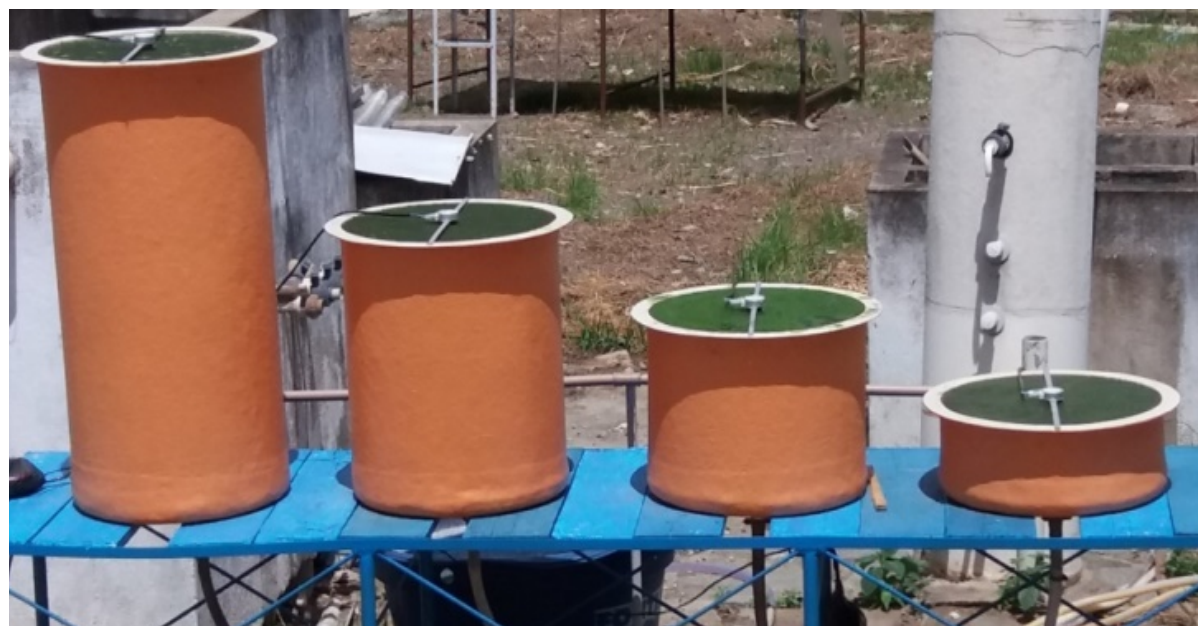

Figura 2 - Imagem real das lagoas em operação. 


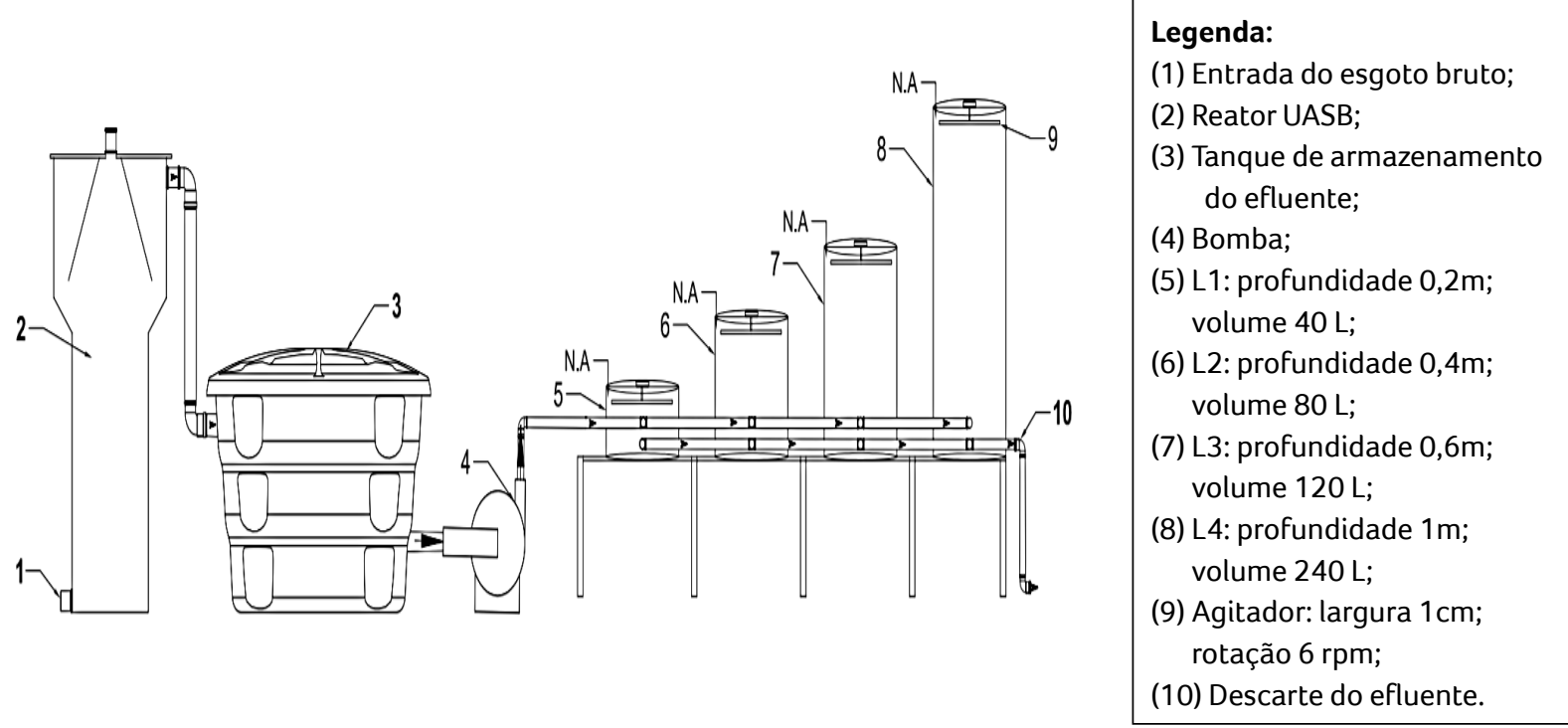

Figura 3 - Desenho esquemático do sistema experimental.

\subsection{Monitoramento do sistema}

A investigação experimental foi dividida em duas etapas: (i) a primeira foi realizada durante oito meses, no período de verão (outubro de 2018 a maio de 2019), cuja temperatura máxima foi de $32^{\circ} \mathrm{C}$ e a mínima de $21^{\circ} \mathrm{C}$; (ii) a segunda etapa foi realizada por três meses no período de inverno (maio a julho 2019), com média de $19^{\circ} \mathrm{C}$ para a temperatura mínima e $27^{\circ} \mathrm{C}$ para a máxima (INMET, 2019). O horário de coleta das amostras foi fixado às 9 horas para ambas as condições. Inicialmente, para melhor acompanhamento do comportamento das lagoas ao longo da batelada, as análises eram realizadas diariamente. Após três meses de monitoramento, a periodicidade das análises foi reduzida para três vezes por semana. Os totais de bateladas das lagoas foram os seguintes:

- L1: 45 bateladas no período de verão e 11 bateladas no período de inverno;

- L2: 30 bateladas no período de verão e 5 bateladas no período de inverno;
- L3: 10 bateladas no período de verão e 3 bateladas no período de inverno;

- L4: 8 bateladas no período de verão e 3 bateladas no período de inverno.

Para a avaliação da qualidade do esgoto antes e após o tratamento, bem como para a verificação da relação de alguns parâmetros, como a remoção de fosfato, foram analisados os parâmetros: $\mathrm{pH}$, oxigênio dissolvido, temperatura, demanda química de oxigênio (DQO), demanda bioquímica de oxigênio (DBO), sólidos totais, alcalinidade e ortofosfato. As metodologias utilizadas seguiram os procedimentos descritos no Standard Methods for Examination of Water e Wastewater (APHA; AWA; WEF, 2017), exceto para a alcalinidade total, que foi realizada de acordo com o método de Kapp (BUCHAUER, 1998).

\section{RESULTADOS E DISCUSSÃO}

O clima do Nordeste brasileiro, onde se localiza a estação experimental (Extrabes), local de realização dos experimentos, é caracterizado por 
apresentar dois períodos distintos: um chuvoso e outro de estiagem, visto que não se observam mudanças relevantes de temperatura e de outras variáveis meteorológicas ao longo do ano. Os períodos com maior índice de precipitação são registrados nos meses de maio a julho, enquanto os meses de outubro a março apresentam baixos índices pluviométricos (INMET, 2019). As temperaturas do líquido nas lagoas variaram entre $20^{\circ} \mathrm{C}$ e $31^{\circ} \mathrm{C}$.

$\mathrm{Na}$ Tabela 2, podem-se observar as concentrações médias das características físico-químicas do esgoto bruto da cidade de Campina Grande e do efluente do reator UASB, que era usado como afluente das lagoas de polimento.
Nas Fig. 4 e 5 foram plotados os valores médios do $\mathrm{pH}$ e da concentração de fosfato nas lagoas em função do tempo das bateladas para o período de insolação máxima (verão) e do período com chuvas e menos insolação (inverno). Estabeleceu-se que as concentrações de fosfato em amostras filtradas e em amostras brutas depois de serem digeridas eram praticamente iguais e passou-se a usar amostras filtradas para os testes de fosfato. Também ficou estabelecido que a concentração de fósforo incorporado nas algas (sólidos em suspensão) era menos que 0,5 mgP.L $\mathrm{L}^{-1}$, de modo que a diminuição da concentração de fosfato na Fig. 5 pôde ser atribuída à precipitação.

Tabela 2 - Caracterização do esgoto bruto (valores médios) e do efluente do reator UASB durante o verão (2018/19) e o inverno (2019).

\begin{tabular}{|c|c|c|c|c|c|}
\hline \multirow{2}{*}{ Variável } & \multirow{2}{*}{ Unidade } & \multicolumn{2}{|c|}{ Verão 18/19 } & \multicolumn{2}{|c|}{ Inverno 19} \\
\hline & & Esgoto bruto & Efluente UASB & Esgoto bruto & Efluente UASB \\
\hline DBO & $\mathrm{mg} \cdot \mathrm{L}^{-1}$ & 378 & 104 & 242 & 99 \\
\hline DQO & $\mathrm{mg} \cdot \mathrm{L}^{-1}$ & 556 & 161 & 546 & 192 \\
\hline SST & $\mathrm{mg} \cdot \mathrm{L}^{-1}$ & 291 & 109 & 172 & 83 \\
\hline Amônia & $\mathrm{mgN} \cdot \mathrm{L}^{-1}$ & 56 & 61 & 28 & 30 \\
\hline P & mgP. $\mathrm{L}^{-1}$ & 6,0 & 7,6 & 4,3 & 5,2 \\
\hline $\mathrm{pH}$ & - & 7,3 & 7,3 & 7,3 & 7,2 \\
\hline Alcalinidade & $\mathrm{mgCaCO}_{3} \mathrm{~L}^{-1}$ & 369 & 438 & 361 & 412 \\
\hline
\end{tabular}
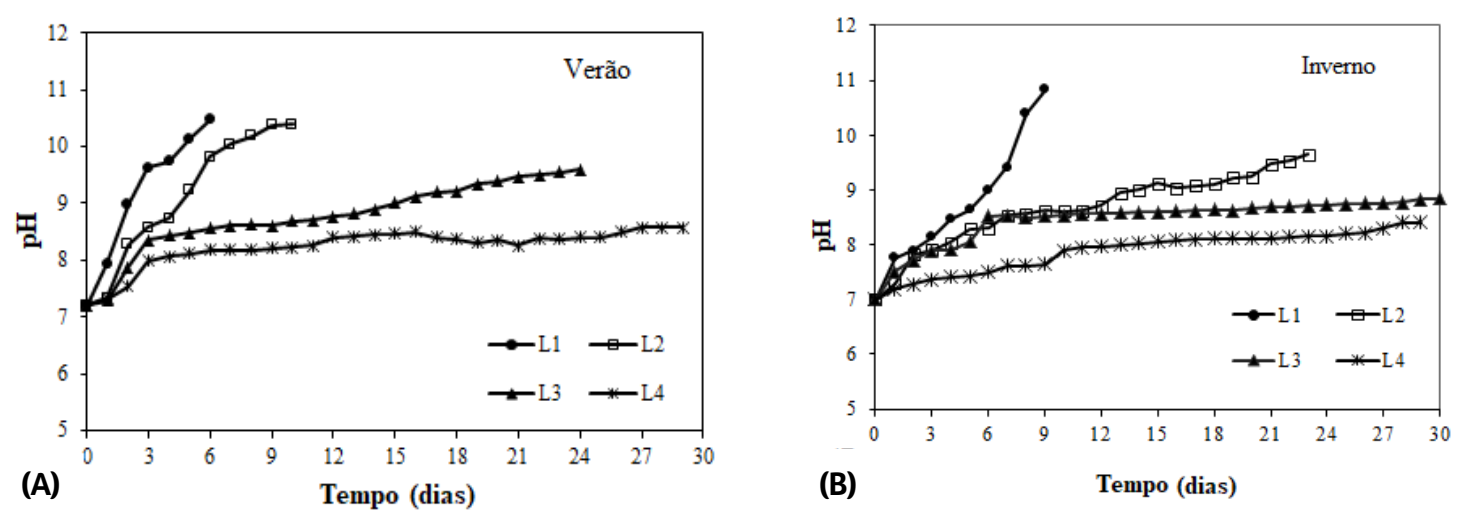

Figura 4 -Variação do pH em função do tempo para as diferentes profundidades durante o verão(A) e durante o inverno (B). 

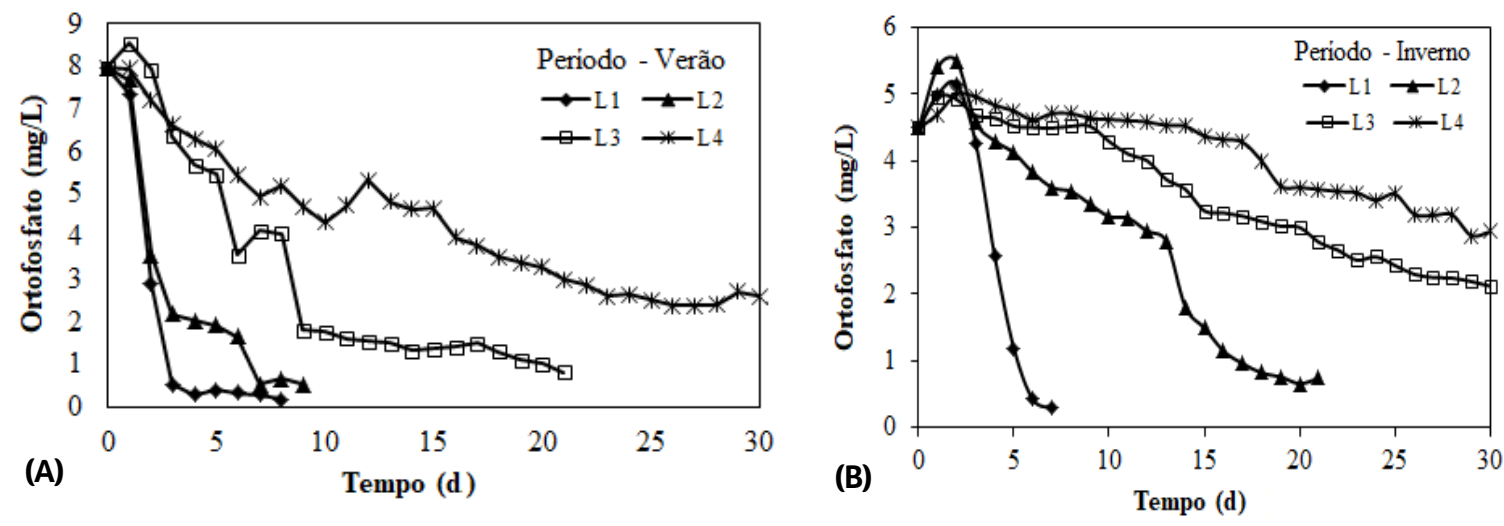

Figura 5 - Variação da concentração de fosfato em função do tempo para as diferentes profundidades durante o verão (A) e durante o inverno (B).

Nas Fig. 4 e 5, a relação entre o aumento do pH e a diminuição da concentração de $\mathrm{P}$ é evidente. Sabendo-se que é necessário um $\mathrm{pH}$ de no mínimo 9 para obter remoção parcial de fosfato por precipitação, pode-se visualizar na Fig. 4A que no período quente esse valor de $\mathrm{pH}$ é atingido em 3, 6, 15 e >30 dias, em L1, L2, L3 e L4, respectivamente, enquanto no período chuvoso (Fig. 4B) esse valor só foi atingido nas lagoas mais rasas, $\mathrm{L} 1 \mathrm{e} \mathrm{L2}$, em um tempo de 5 e 15 dias, respectivamente.

Comparando a concentração de fosfato nos períodos de verão e inverno, nota-se uma nítida diferença: o aumento do $\mathrm{pH}$ e a consequente diminuição da concentração de fosfato é bem mais rápido no período de verão, o que pode ser atribuído tanto à maior intensidade da radiação solar quanto ao aumento da temperatura. A temperatura mais alta no verão leva a uma aceleração do crescimento das algas, o que induz a uma maior taxa de fotossíntese e o aumento mais rápido do $\mathrm{pH}$.

A lagoa mais rasa $L 1(0,20 \mathrm{~m})$, por ser a que permite maior penetração dos raios solares em toda a profundidade, foi altamente eficiente na remoção do fosfato, apresentando concentrações abaixo de $1 \mathrm{mgP.} \mathrm{L}^{-1}$ em aproximadamente 4 dias no período quente e 8 dias no período chuvoso. A L2 $(0,40 \mathrm{~m})$ apresentou comportamento semelhante à $L 1$, mostrando-se capaz de obter um efluente com concentrações menores do que $1 \mathrm{mgP} . \mathrm{L}^{-1}$ em 8 dias de operação da batelada no período quente, enquanto no período chuvoso foi necessário o dobro do tempo. Em relação à $L 3$ $(0,60 \mathrm{~m})$, no período quente foram necessários aproximadamente 18 dias para que a concentração de $P$ nessa lagoa atingisse valores menores do que $1 \mathrm{mgP} . \mathrm{L}^{-1}$, enquanto no inverno não foi possível obter essa concentração no espaço de tempo analisado de 30 dias. A concentração mínima atingida na $L 3$ no período de 30 dias durante o clima não favorável foi de 1,9 mgP..-1 .

Nota-se que o aumento do $\mathrm{pH}$ na lagoa de maior profundidade (L4) é mais demorado. Embora essa lagoa fosse operada durante 30 dias, ainda assim não alcançou valores de $\mathrm{pH}$ acima de 9 , em ambos períodos analisados, e não produziu efeitos significativos em relação à remoção de fósforo. As condições dentro da lagoa não foram favoráveis ao maior aumento do $\mathrm{pH}$, visto que a profundidade de $1 \mathrm{~m}$ não permitiu que a incidência de radiação solar na lagoa fosse aproveitada para a fotossíntese na parte inferior desta e, consequentemente, o aumento do $\mathrm{pH}$ era lento.

Chega-se à conclusão de que a remoção biológica de fósforo não é factível em lagoas de polimento com profundidades maiores do que $1 \mathrm{~m}$, nem mesmo quando existem condições favoráveis ao aumento do $\mathrm{pH}$, como a intensa radiação 
solar e a elevada temperatura obtida no período de verão. Portanto, a remoção de fósforo em LPBS é opcional: viável em lagoas rasas e não factível em lagoas profundas. Se o efluente for direcionado para o reúso agrícola, convém utilizar lagoas mais profundas para reter o fosfato presente e aplicá-lo como fertilizante. Para os casos em que o efluente tratado será descarregado em águas superficiais, é necessária a aplicação de lagoas mais rasas para remover ao máximo a concentração de fósforo (e também nitrogênio, que requer um $\mathrm{pH}$ mais baixo) e assim obter um efluente que atenda aos padrões de lançamento estabelecidos pela legislação ambiental, evitan- do o processo de eutrofização e deterioração da qualidade da água.

Procurou-se estabelecer qual foi o composto de fosfato que precipitou nas LPBS, sabendo-se que a concentração de cálcio no efluente tratado era de aproximadamente $1 \mathrm{mmol} . \mathrm{L}^{-1}$. Para tanto, plotaram-se os valores experimentais da concentração de fosfato em função do $\mathrm{pH}$ para testes nas diferentes profundidades e para o período de verão (Fig. 6) e de inverno (Fig. 7). Assim, esses dados experimentais puderam ser comparados com as curvas da solubilidade termodinâmica de fosfato traçadas na Fig. 1.
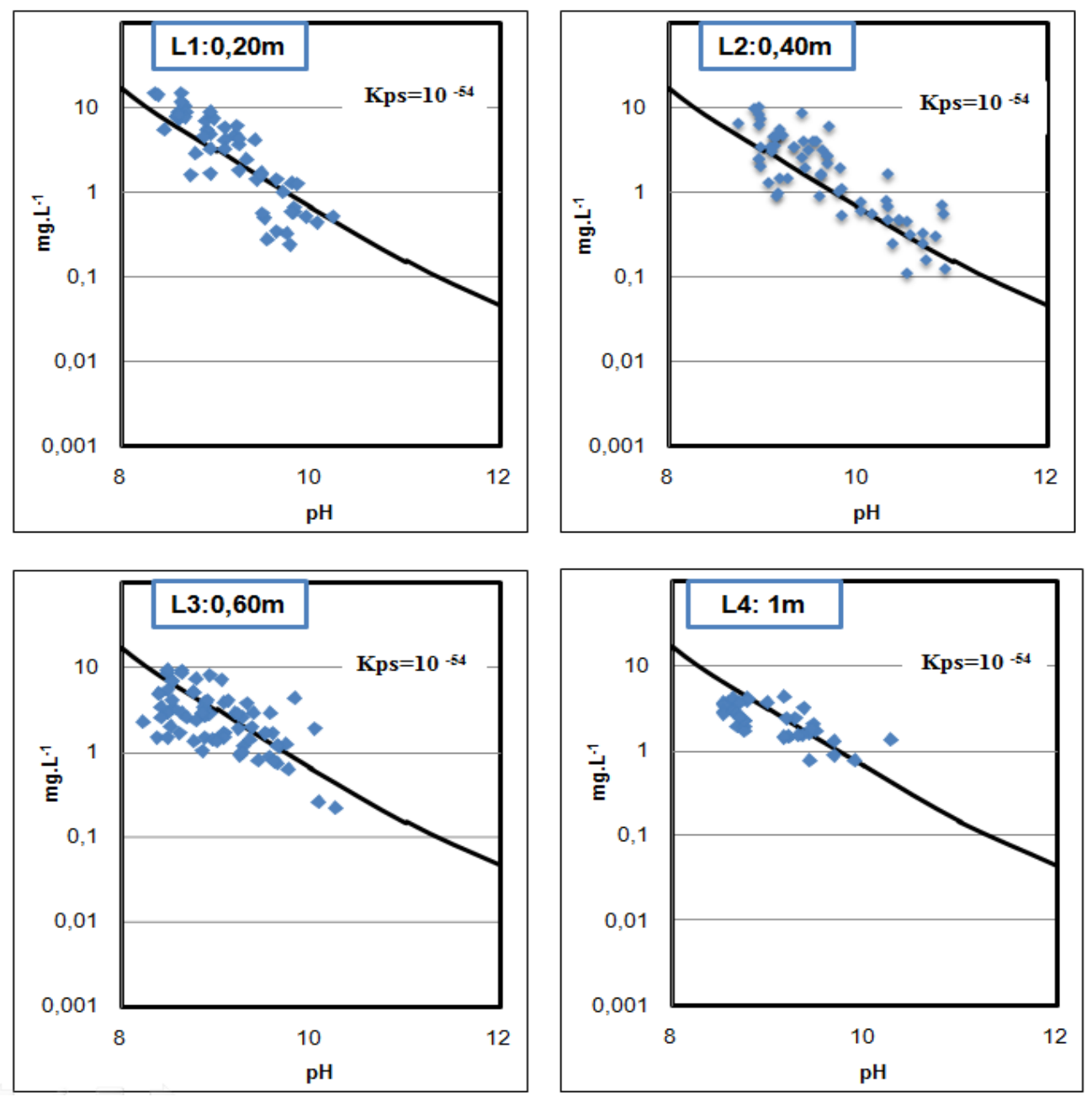

Figura 6 - Dados experimentais de fosfato em função do pH e curva de solubilidade de fosfato para uma constante de solubilidade aparente de $1 \times 10^{-54}$ e uma concentração de cálcio de 1 mmol/L (dados de verão de 2018/2019). 

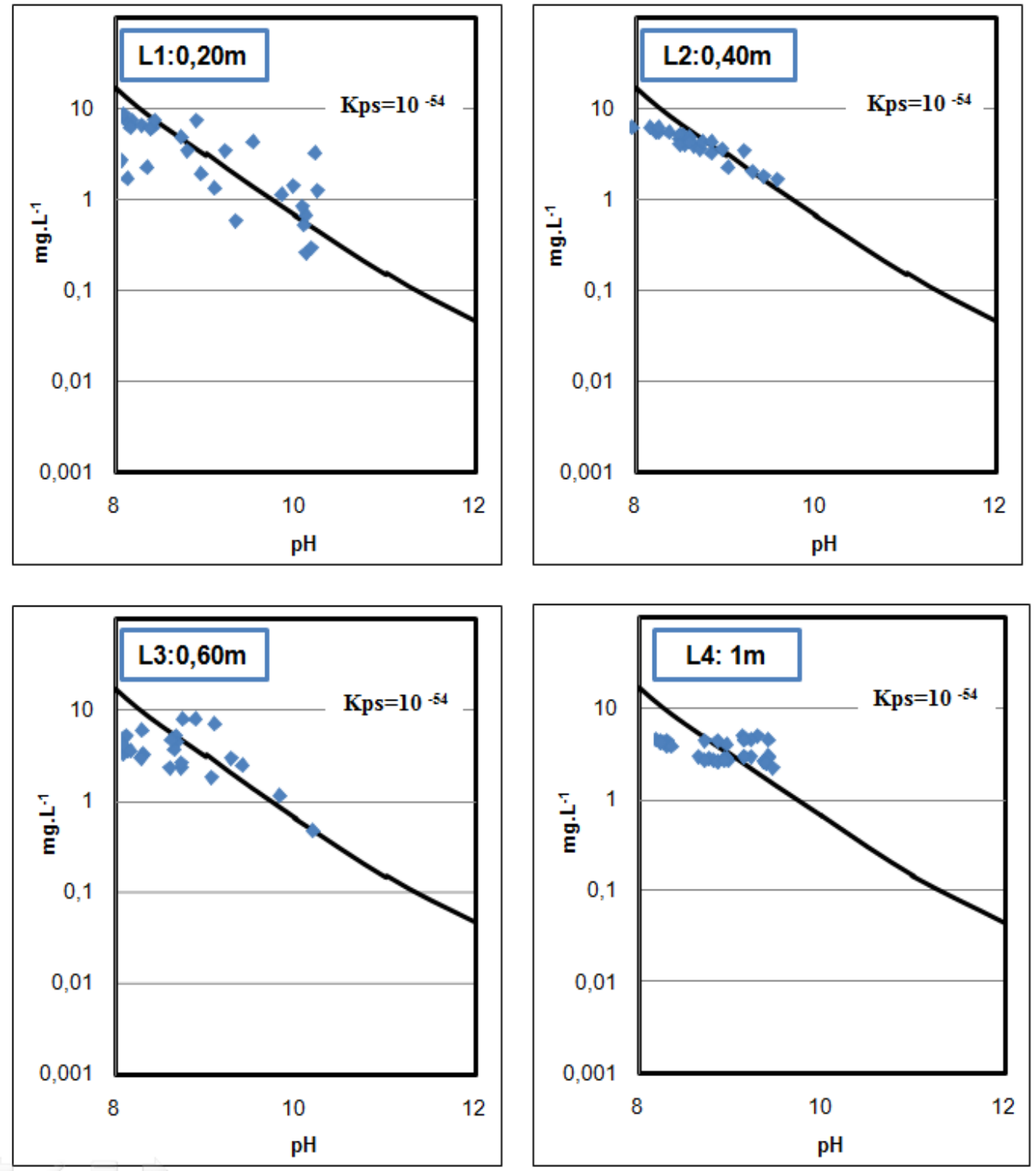

Figura 7 - Dados experimentais de fosfato em função do pH e curva de solubilidade de fosfato para uma constante de solubilidade aparente de $1 \times 10^{-54}$ e uma concentração de cálcio de $1 \mathrm{mmol} / \mathrm{L}$ (dados de inverno, maio a julho de 2019 ).

Observa-se que o comportamento dos dados experimentais se assemelha ao das curvas de solubilidade teóricas da Fig. 1, nos quais, à medida em que o $\mathrm{pH}$ se torna mais alcalino, o fosfato se torna cada vez mais insolúvel. Entretanto, esses sais de fosfato apresentados possuem solubilidade termodinâmica menor do que os valores obtidos experimentalmente. Todavia, quando se aumenta a constante de solubilidade do composto hidroxiapatita de cálcio $\mathrm{Ca}_{10}(\mathrm{OH})^{2}\left(\mathrm{PO}_{4}\right)^{6}$ de
$1 \times 10^{-58,5}$ para $1 \times 10^{-54}$, os valores experimentais e teóricos da concentração de fosfato em função do $\mathrm{pH}$ exibem uma boa correlação para todo o conjunto de dados experimentais, para qualquer uma das quatro profundidades das lagoas, seja no período de verão ou no de inverno. No entanto, a necessidade de alterar o valor da constante de solubilidade significa dizer que a precipitação de fosfato não foi completa: em vez de alcançar o valor termodinâmico de $10^{-58,5}$, a precipitação 
cessou quando a concentração de fosfato alcançou um valor que combina com uma constante aparente de solubilidade de $1 \times 10^{-54}$. Isso mostra que o produto de solubilidade do fosfato na lagoa era maior no esgoto do que o valor termodinâmico, para qualquer valor de $\mathrm{pH}$. A maior solubilidade de fosfato em efluentes, especialmente os que contêm material orgânico, já foi observada em estudo anterior (CAPRI; MARAIS, 1974). Esses resultados vão de encontro àqueles obtidos por House (1999) e Castro et al., (2012 e2013), os quais relataram que na faixa de $\mathrm{pH}$ de 9 a 12,0, a precipitação de HA predomina sobre as outras formas de fosfatos de cálcio.

Os dados experimentais mostram ainda que é possível obter uma concentração baixa de fosfato ( $\left.1 \mathrm{mgP}^{-1} \mathrm{~L}^{-1}\right)$, mas o tempo de permanência na LPBS é influenciado pelo clima e pela profundidade das LPBS. O período é relativamente curto para lagoas rasas no verão. No inverno, o tempo necessário é bem maior (em torno do dobro) para todas as profundidades. Para LPBS profundas (> $1 \mathrm{~m}$ ), a remoção de $P$ se torna impraticável, visto que o tempo de permanência se torna excessivo. $\mathrm{Na}$ verdade, essas lagoas são indicadas se o objetivo do tratamento é preservar o fósforo.

Assim, com base na profundidade da lagoa de polimento, pode-se optar por remover ou não o fosfato. Na condição do semiárido brasileiro, possivelmente se aplicariam lagoas profundas no verão para reter o fosfato e usar o efluente para reúso agrícola. No inverno, caso houvesse bastante chuva, possivelmente não se praticaria o reúso e o efluente final seria descarregado em águas de superfície. Nesse caso se aplicaria uma lagoa de profundidade da ordem de 0,4 metros para precipitar o fosfato.

Uma variável que também deve ser levada em consideração para o emprego de lagoas de polimento é a área per capita. Essa área está relacionada com a profundidade das lagoas e com o tempo de detenção hidráulica. A área necessária para a construção das lagoas de polimento pode ser calculada por meio da seguinte formulação (MARA et al., 1992):

$A=T D H \times Q P H$

Em que:

$A=$ área requerida para LPBS $\left(\mathrm{m}^{2}\right.$. habitante $\left.{ }^{-1}\right)$;

TDH = tempo de detenção hidráulica (dias);

$\mathrm{Q}$ = vazão diária do efluente $\left(\mathrm{m}^{3}\right.$. habitante $\left.\mathrm{h}^{-1} \cdot \mathrm{dia}^{-1}\right)$;

$\mathrm{H}=$ profundidade da lagoa $(\mathrm{m})$.

Na Tabela 3 é apresentado o TDH observado para que as lagoas com diferentes profundidades atingissem um efluente com a concentração de aproximadamente 1 e $2 \mathrm{mgP.L}^{-1}$ (Coluna 2 e 3). Nas colunas 4 e 5, pode-se observar os valores da área per capita para as concentrações residuais de 1 e 2 mgP.L ${ }^{-1}$, calculadas com a Eq. 9 para uma contribuição per capita de $0,1 \mathrm{~m}^{3} \cdot \mathrm{d}^{-1}$ para o período de verão. 0 cálculo para a obtenção da área no período com maior precipitação não foi realizado, visto que o TDH no inverno é bem mais elevado. Esse aspecto deve ser considerado, uma vez que nem sempre será viável a utilização dessas lagoas em regiões com clima frio. Nas lagoas rasas (0,2 a 0,4 $\mathrm{m}$ de profundidade), a área necessária é bem menor do que em lagoas de estabilização convencionais, onde a área é da ordem de $3 \mathrm{~m}^{2} / \mathrm{hab}$, sendo que nestas a remoção de fósforo não é possível. Portanto, se a remoção de fósforo é desejada, a combinação de UASB-LPBS com lagoas rasas é muito superior a lagoas de estabilização convencionais.

Uma possibilidade para remoção de fósforo em condições climáticas desfavoráveis é a aplicação da clarificação, usando sais trivalentes como o sulfato de alumínio ou cloreto férrico. A clarificação provoca a precipitação quase total do fósforo. Portanto, se a clarificação for prevista para o efluente das LPBS, a remoção de fósforo por elevação do $\mathrm{pH}$ não é necessária; precisa-se somen- 
te remover o nitrogênio amoniacal, e para tanto o tempo de permanência e a área per capita são muito menores, em torno da metade do valor necessário para a precipitação de fosfato sem coagulante. Essa é uma opção quando se preten- de aplicar o efluente final para reúso industrial, pois nesse caso é necessário que se removam todos os sólidos em suspensão para produzir um efluente com boa qualidade e baixa turbidez, o que é factível pela aplicação da clarificação.

Tabela 3 - Tempo de detenção hidráulica necessário para operação das LPBS

\begin{tabular}{|c|c|c|c|c|}
\hline 1 & 2 & 3 & 4 & 5 \\
\hline $\begin{array}{l}\text { Profundidade } \\
\text { da lagoa }\end{array}$ & $\begin{array}{l}\text { TDH - para } \\
2 \text { mgP.L }{ }^{-1}\end{array}$ & $\begin{array}{c}\text { TDH - para } \\
1 \mathrm{mgP}^{-\mathrm{L}^{-1}}\end{array}$ & $\begin{array}{l}\text { Área per capita para } \\
\qquad 2 \mathrm{mgP} \cdot \mathrm{L}^{-1}\end{array}$ & $\begin{array}{c}\text { Área per capita para } \\
1 \mathrm{mgP} . \mathrm{L}^{-1}\end{array}$ \\
\hline $\mathrm{L} 1=0,2 \mathrm{~m}$ & 3 dias & 4 dias & 1,5 & 2 \\
\hline $\mathrm{L} 2=0,4 \mathrm{~m}$ & 6 dias & 8 dias & 1,5 & 2 \\
\hline $\mathrm{L} 3=0,6 \mathrm{~m}$ & 15 dias & 21 dias & 2,5 & 3,5 \\
\hline$L 4=1,0 \mathrm{~m}$ & 30 dias & Estimado 40 dias & 3 & 4 \\
\hline
\end{tabular}

\section{CONCLUSÕES}

A remoção biológica de fósforo é factível em lagoas de polimento operadas em regime de bateladas sequenciais. A remoção eficiente e confiável de fósforo em lagoas de polimento está associada ao aumento do $\mathrm{pH}$, que por sua vez depende principalmente de dois fatores: (1) alta taxa de consumo de $\mathrm{CO}_{2}$, ou seja, a taxa de fotossíntese na lagoa deve ser superior à taxa de produção de $\mathrm{CO}_{2}$ pela oxidação de material orgânico; e (2) baixas profundidades, pois quanto menor a profundidade da lagoa, maior será a taxa de fotossíntese e, portanto, o aumento do $\mathrm{pH}$.

O tempo de permanência nas lagoas de polimento para a remoção de fósforo é mais curto no período de alta intensidade de radiação solar, principalmente nas lagoas de menor profundidade, onde foi possível obter valores de $\mathrm{pH}$ acima de 9,5 e efluentes com concentrações de fósforo inferiores a $1 \mathrm{mgP.L} \mathrm{L}^{-1}$.

Os dados experimentais indicam que o fosfato precipitou como hidroxiapatita de cálcio nas lagoas, no entanto a solubilidade experimental nas lagoas foi maior do que a solubilidade termodinâmica.

Se o reúso agrícola é previsto para o efluente final, a remoção de fósforo deve ser evitada, o que pode ser feito pela escolha de uma profundidade suficiente para evitar um aumento do $\mathrm{pH}$ (profundidade $\geq 1 \mathrm{~m}$ ). Nos casos em que o efluente será lançado em corpos de água, a remoção do fósforo é necessária para evitar o processo de eutrofização e a deterioração da qualidade da água; nesse caso, a profundidade da lagoa será pequena $(0,2$ a $0,6 \mathrm{~m})$.

Quando se prevê reúso industrial do esgoto tratado, é necessário que se removam os sólidos em suspensão, por exemplo, por clarificação. Nesse caso, a remoção de fosfato será realizada pelo uso de coagulantes e a precipitação nas lagoas não é necessária. Sob essas condições, o tempo de permanência e a área necessária para a construção das lagoas, serão determinados pela necessidade de remover nitrogênio.

\section{AGRADECIMENTOS}

Os autores agradecem ao Conselho Nacional de Desenvolvimento Científico e Tecnológico (CNPq) pelo incentivo financeiro as pesquisas.

\section{CONTRIBUIÇÃO DOS AUTORES}

Todos os autores contribuíram de forma igualitária. 


\section{REFERÊNCIAS}

AMERICAN PUBLIC HEALTH ASSOCIATION - APHA; AMERICAN WATER WORKS ASSOCIATION - AWWA; WATER ENVIRONMENT ASSOCIATION - WEF. Standard Methods for the Examination of Water and Wastewater. 23. ed. Washington D. C, 2017.

ALBUQUERQUE, M. S.; SANTOS, S. L.; VAN HAANDEL, A. Influência do regime hidrodinâmico sobre o desempenho de lagoas de polimento. Revista DAE: Edição especial, São Paulo, n. 229, Março, 2021.

BASTOS, R. K. X.; RIOS, E. N.; DORNELAS, F. L.; ASSUNÇÃO, F. A. L.; NASCIMENTO, L. E. Ammonia and phosphorus removal in polishing ponds: A case study in Southeast Brazil. Water Science and Technology. v. 55, n.11, p. 65-71, 2007.

BRASIL. Ministério do Meio Ambiente. CONAMA. Resolução $n^{\circ}$ 430, de 13 de maio de 2011: Dispõe sobre as condições e padrões de lançamento de efluentes, complementa e altera a Resolução $n^{\circ}$ 357, de 17 de março de 2005, do Conselho Nacional do Meio Ambiente CONAMA. Diário Oficial da União, Brasília, 2011.

BRASIL. Ministério do Meio Ambiente. CONAMA. Resolução $\mathrm{n}^{\circ}$ 357, de 17 de março de 2005: Dispõe sobre a classificação dos corpos de água e diretrizes ambientais para o seu enquadramento, bem como estabelece as condições e padrões de lançamento de efluentes, e dá outras providências. Diário Oficial da União, Brasília, 2005.

BUCHAUER, K. A comparison of two simple titration procedures to determine volatile fatty acids in influents to waste-water and sludge treatment processes. Water Sa-Pretoria, v. 24, p. 49-56, 1998.

CAPRI, M. G.; MARAIS, G. V. R. Anaerobic Filter Treatment of Wine Distillery Waste, Research Report W1, Dep. of Civil Engineering, University of Cape Town, África do Sul, 1974

CASAROTTI, E. G.; MATSUMOTO, T.; ALBERTIN, L. L. Evaluation of the wind influence in the circulation of a stabilization pond. Engenharia Sanitária e Ambiental, 17(4), 401-412, 2012.

CASTRO, F.; FERREIRA, A.; ROCHA, F.; VICENTE, A.; E TEIXEIRA, J. A. Characterization of intermediate stages in the precipitation of hydroxyapatite at $37 \mathrm{C}$. Chemical engineering science, v. 77, p. 150-156, 2012.
CASTRO, F.; KUHN, S., JENSEN, K.; FERREIRA, A.; ROCHA, F., VICENTE, A.; TEIXEIRA, J. A. Continuous-flow precipitation of hydroxyapatite in ultrasonic microsystems. Chemical engineering journal, v. 215, p. 979-987, 2013.

CAVALCANTI, P. F. F. Integrated Application of the UASB Reactor and Ponds for Domestic Sewage Treatment in Tropical Regions. (PhD thesis) - University of Wageningen, The Netherlands, 2003.

CHOW, L. C.; EANES, E. D. Solubility of calcium phosphates. Monographs in oral science, 18, 94-111, 2001.

HOUSE, W. A. The Physico-Chemical Conditions for the Precipitation of Phosphate with Calcium. Environ Technol. 20, 727-733. 1999.

INSTITUTO NACIONAL DE METEOROLOGIA - INMET. Estações Automáticas. Disponível:<http://www.inmet.gov.br/portal/index. php?r=estacoes/estacoesAutomaticas $>$. Acesso: 25.07.2019

JORDÃO, E.P.; PESSÔA, C.A. Tratamento de esgotos domésticos. 8.ed. Rio de Janeiro: ABES, 2017

MARA, D. D.; ALABASTER, G. P.; PEARSON, H. W.; MILLS, S. W. Waste stabilization ponds: a design manual for Eastern Africa. Lagoon Technology International, Leeds. 1992

MORAIS, C. E. P; SANTOS, S. L.; VAN HAANDEL, A. Variação do pH e remoção de nitrogênio em lagoas de polimento. Revista DAE: Edição especial, São Paulo, n. 229, Março, 2021.

PARKER, C. D.; JONES, H. L.; TAYLOR, W. S. Purification of Sewage in Lagoons. Sewage and Industrial Waste, v. 22, n. 6, p. 760-775, 1950. https://doi.org/10.2307/25031317

REED, S.C.; CRITES, R.W.; MIDDLEBROOKS, E.J. Natural systems for waste management and treatment. $2 \mathrm{Ed}$. New YorK, USA: McGraw-Hill Inc., 1995. 433p. 1995.

VAN HAANDEL, A. C.; LETTINGA, G. Anaerobic sewage treatment: a practical guide for regions with a hot climate. John Wiley and Sons, 1994, 222p.

VON SPERLING, M., CARAUCCI FILHO, B., MONTEGGIA, L., \& PIVELLI, R. Remoção de Nutrientes em Sistemas Naturais. In: Nutrientes de esgoto sanitário: utilização e remoção. MOTA, F.S.B.; VON SPERLING, M. Projeto PROSAB. Rio de Janeiro: ABES, 2009. 428p. 